\title{
El pensamiento de José María Izquierdo Arroyo en la organización y representación del conocimiento: una sistematización desde fundamentos filosóficos y semióticos
}

José María Izquierdo Arroyo's thinking in knowledge organization and representation: a systematization on philosophical and semiotic foundations

\section{Luis Miguel MoReno FeRnÁndez (1), Mónica IZQUIERdo Alonso (2)}

(1) Universidad de Murcia, morferdez@um.es. (2) Universidad de Alcalá, monica.izquierdo@uah.es

\begin{abstract}
Resumen
Se analizan las aportaciones científicas de José María Izquierdo a la lingüística y semiótica documental, a partir de una selección y comentario de su obra. Desde posicionamientos claramente lógico-formalistas, a la vez que lingüísticos, revisamos su concepción de la organización y representación del conocimiento, desde tres núcleos de acción: a) trabajos centrados en la teoría de documentación, b) estudios orientados hacia la teoría de la comunicación y la semiología, y c) investigaciones relacionadas con la lingüística documental y semiótica documental: teoría del análisis lógico-lingüístico, gestión de lenguajes documentales y sistemas automáticos de procesamiento del discurso. Finalmente, se alude al legado doctrinal del autor en su grupo de investigación, desde la concepción del esquema semiótico como motor de las investigaciones, enfocado éste hacia el resumen documental, la pragmática en la representación documental, y la lingüística descriptiva y comparada de los lenguajes documentales.
\end{abstract}

Palabras clave: Semiótica documental. Lingüística documental. Organización del conocimiento. Representación documental. Epistemología. José María Izquierdo Arroyo.

\section{Introducción}

Filósofo, profesor, investigador y poeta -faceta ésta última que muy pocos conocen-, su sólida formación académica en filosofía y letras (con importantes desarrollos en lógica formal, filosofía de la ciencia y filosofía del lenguaje), junto con un sistemático autodidactismo filológico (cimentado en el estructuralismo y el funcionalismo lingüístico), se traslucirá en cada uno de sus trabajos y le permitirá establecer oportunas conexiones entre la fenomenología, la semiología, el pragmatismo, las gramáticas discursivas, la teoría del conocimiento y los sistemas de organización y representación documental. Este rico sustrato académico lógico-lingüístico será determinante a la hora de concebir su teoría de

\begin{abstract}
This work considers the scientific contributions of José María Izquierdo Arroyo to Knowledge Organization and Representation. From a logic-linguistic point of view, three outbreaks of research are discussed: studies based on the theory of Information Science, Communication and Semiology and Information Science Linguistics. Finally, a reflection is done about the influence of the aforementioned author in his research group from an information scicen semiotics point of view.
\end{abstract}

Keywords: Information Science Semiotics. Information Science Linguistics. Knowledge Organization. Knowledge Representation. Epistemology. José María Izquierdo Arroyo.

Sólo soy un tejedor que está aprendiendo el oficio; me prestaron el telar, y mis hilos son los libros...

(Izarro, 1979)

la documentación, el concepto extendido de documento y el de representación/lenguaje documental, tomando como referente el paraguas de la semiótica documental y los modelos de la lógica formal (simbólica y matemática).

Tras su publicación en 1980 de su obra Sobre la transducción, meditaciones semiológicas, y desde sus primeras reflexiones a inicios de los 90 relacionadas con el tratamiento documental de contenido y el tratamiento temático de la información, formuló y conceptualizó, en el marco de una semiótica estructural-funcional, su propuesta de semiótica documental y definió sus modelos formales para la representación documental y el estudio de los lenguajes documentales. 
Desde sus primeras incursiones en el área documental, y especialmente en la organización y representación del conocimiento, destacamos tres itinerarios epistemológicos de ruta:

- La concepción del documento (soporte, objeto, lenguaje, pensamiento y acción) como recurso no sólo epistemológico sino también como fenómeno pragmático documental, revisando su documentabilidad desde procesos de semiosis social y heurística, a la luz de la ciencia de la búsqueda secundaria: la Documentación y sus procesos de análisis.

- El posicionamiento ante una nueva perspectiva, la semiótica documental, dentro de un campo del saber fuertemente institucionalizado por aquel entonces con arraigada tradición francófona, la lingüística documental. En este punto, subrayamos la apuesta edificadora de Izquierdo con una rica sistematización teórica y metodológica, a partir de la combinación de teorías y conceptos lingüísticosemióticos y filosóficos, entre otros.

- Una detallada revisión y adaptación crítica de los modelos estructural-funcionalistas, empapados de una fuerte base formalista heredada del legado lógico, aplicados a los métodos de tratamiento documental de contenido y a las técnicas de representación y procesamiento del discurso.

\section{Diálogos teóricos y aportaciones científicas}

En este epígrafe realizaremos una revisión de sus trabajos, analizando con especial cuidado las obras que jalonan su fecundo discurso teórico. Nos detendremos en sus áreas de actividad más definidas con el objetivo de revisar y destacar los conceptos nucleares que constituyen su línea de pensamiento y sus principales aportaciones en el ámbito de la información-documentación, especialmente en lo que respecta a la organización y representación documental del conocimiento.

Es difícil establecer periodizaciones para un pensamiento continuo y cíclico ya que muchos de los conceptos que constituyen la impronta de Izquierdo son transversales en toda su obra. Por ello, al segmentar el corpus teórico para nuestro estudio, no siempre atendemos a razones cronológicas sino más bien funcionales y temáticas. De este modo, abordamos el pensamiento de este autor desde un diálogo-interpretación atendiendo a tres ejes de acción científica:

- Estudios centrados en la teoría de la documentación: a) teoría general de la documen- tación y b) estudios monográficos sobre documentación.

- Teoría de la comunicación y semiología

- Investigaciones relacionadas con la semiótica documental. Trabajos enfocados hacia la teoría del análisis lógico-lingüístico, gestión de lenguajes documentales y sistemas automáticos de procesamiento del discurso.

\subsection{Aportaciones a la consolidación}

la documentación como ciencia: teoría general de la documentación o ciencia de la información

Recogemos aquí una serie de investigaciones que suponen un marco epistemológico para la consolidación de la ciencia de la documentación. En este contexto, diferenciamos dos núcleos de aportaciones: a) uno enfocado hacia la constitución de una teoría general de la documentación, en un momento en el que la disciplina se constituye como tal en nuestro país (finales de 1970 e inicios de 1980) y b) una segunda vertiente de estudios monográficos, con trabajos más específicos sobre distintos aspectos de la ciencia de la documentación.

\subsubsection{Teoría general de la documentación}

A la fundamentación disciplinar de la Documentación pertenecen el libro Concepción lógicolingüística de la Documentación (1983) y el artículo Análisis formal de las definiciones de Documentación (1990). A este grupo temático se adscriben los artículos La Ciencia de la Búsqueda Secundaria l: razón y misión de la CBS (1989) y Problemas y perspectivas en la comunicación del saber (1989).

Concepción Lógico-Lingüística de la Documentación (1983)

Es una monografía básica para la conceptualización de la documentación que ha abierto nuevos surcos dentro del área. Asienta las bases pragmáticas, teóricas y técnicas de la documentación, con seguimiento histórico que recorre la fase preotletiana, su actual estado como «ciencia normal» y la propuesta, por parte de Izquierdo, de un nuevo paradigma, radicado en su «extensión», mediante el concepto de interdocumento/archidocumento.

El instrumental utilizado son los métodos y técnicas de la lógica y de la lingüística modernas, en dos accesos complementarios: a) el análisis del lenguaje ordinario, centrado en los campos pragmáticos correspondientes a las acciones de "comunicar", "informar" y "documentar"; y b) el estudio de la obra pionera de Paul Otlet. 
Los pilares en que se apoya la construcción del libro pertenecen a tres ámbitos científicos: lógica y filosofía de la ciencia, la teoría del lenguaje y semiótica general y, finalmente, la lingüística positivista. Cabe reseñar la metodología del tratamiento de la microlingüística documental, destacando aspectos relacionados con la teoría de la definición (aplicables actualmente al campo del diseño terminológico) y, más expresamente, los que aluden a la fórmula concepcional del proceso de conceptualización de la documentación (p. 94-98 y p. 300-302). Constituyen éstos los fundamentos, desde fines de los 90 , para los desarrollos en representación del conocimiento basados en ontologías, grafos, redes neuronales y bayesianas, etc. Destaca, asimismo, el análisis de los campos pragmáticos correspondientes a las tres raíces (doc, inform., comu.) (p. 172-258); la aplicación del grafo de procesiones semánticas y el método de los árboles etimológicos para el establecimiento de los campos semánticos de estos términos. Esta tríada, conforma la base metodológica para los nuevos sistemas de representación del conocimiento en el dominio de la gestión de tesauros y ontologías.

En el trabajo se parte de los fundamentos mismos de la teoría de la documentación mediante: el establecimiento de los criterios de conceptualización y de definición; el estudio del sistema científico, y, en particular, y dentro del mimo, el problema de la interdisciplinariedad; y la propuesta de la tipología ciencia/disciplina/asignatura, etc. Este método resultante de la intersección de la lógica y la lingüística se apoya sobre diversos cimientos, entre los que podemos citar los postulados de la Escuela de Oxford, el modelo de Coseriu, las ideas de Pike y la teoría de sistemas sobre la que se ha construido un valiosísimo esquema cibernético-procesual. Sobre todo lo expuesto se basan las dos concepciones de la documentación postuladas en la obra: la ordinaria y la científica, relacionada esta última con la gran aportación de Otlet. Por otra parte, se lleva a cabo un estudio pormenorizado de: a) las denominaciones más adecuadas, prestando especial relieve al término documentología; b) del concepto de documento en sus aspectos lingüísticos, físico, psíquico o intelectual y social; $y$, finalmente, c) del problema de la organización de la documentación.

La Ciencia de la Búsqueda Secundaria I: razón y misión de la CBS (1989)

El objetivo de este estudio es sistematizar los fenómenos históricos y teóricos que dieron origen a la ciencia de la documentación en el marco general del conocimiento científico. Dicho análisis permitió la utilización de la denomina- ción "ciencia de la búsqueda secundaria" para distinguirla de la ciencia convencional de la búsqueda primaria. Como características menos tópicas de este trabajo se han de enumerar: la sistematización de tópicos; la concepción de la ciencia como proceso de búsqueda; la recusación del "acumulativismo" y del "especialismo" científicos; la llamada de atención sobre el profetismo de Ortega y Gasset respecto a nuestra disciplina; y la denuncia de la moda de atender morosamente a lo más reciente, en las búsquedas bibliográficas retrospectivas.

Problemas y perspectivas en la comunicación del saber (1989)

El trabajo, desde un claro posicionamiento filosófico, defiende el problema de la comunicación como núcleo caracterizador de la postmodernidad a partir de una combinatoria secular en que se juega con la terna "haber", "conocer" y "comunicar".

Análisis formal de las definiciones de Documentación (1990)

En este artículo se ahonda en el ámbito epistemológico de la documentación, conceptualizándola y abordando el problema de su definición. Se parte de un corpus definicional de más de 120 definiciones de la ciencia de la documentación sometiéndole a análisis mediante el aparato formal del análisis componencial. Ello le permite al autor dar con una definición inductiva para la documentación. El esquema cibernético procesual de tratamiento analítico se basa en la identificación de cinco facetas o componentes en cada una de las definiciones del concepto: denominación, carácter, materia, proceso y especificación. Con ello, se crea una tabla definicional cuya lectura induce, mediante selección de constantes definicionales, a los que se denomina ciencia "normal" de la documentación.

\subsubsection{Estudios monográficos sobre Documentación.}

Agrupamos bajo este subepígrafe trabajos más específicos sobre distintos aspectos de la ciencia de la documentación.

Reflexiones sobre "documento": palabra / objeto (1982)

A lo largo del estudio, Izquierdo Arroyo fija el concepto de documento en una terminología desambiguada y de contenido preciso. Para ello, aborda el acceso al concepto de documento en dos vertientes: fijación semántica (uso y mención del término) y fijación etimológica. Con este propósito, acomete el problema de la correlación entre definición y denominación, radicándolas en los aspectos semánticos de la sema- 
siología y onomasiología respectivamente. El análisis semántico se realiza sobre la base del estudio específico de varios glosarios y tesauros terminológicos latinos. El semasiológico se lleva a cabo mediante diagramas arbóreos de flujo etimológico-semántico. En el trabajo se avanza una síntesis filológica provisional del concepto de documento y una tipología combinatoriamente abierta a otras instancias ejemplificadoras. El autor se sirve de un esquema definicional en el que las distintas variables constituyentes pueden ser sustituidas uniformemente por diversas acepciones de "documento" (en un procedimiento análogo al de la fórmula concepcional del libro concepción lógico-lingüística). Este acceso filológico al concepto de "documento" habrá de conjugarse con un acceso normal (inducción definicional de constantes) y un acceso constructivo-funcional (en el contexto de un modelo comunicativo-documental).

\section{Cuatro trabajos en curso (1992)}

Son cuatro trabajos especialmente relacionados con la lingüística documental que asientan las bases de la concepción interdisciplinar de la disciplina, especialmente desde las ciencias formales y del lenguaje. La semiótica documental (lingüística documental latu sensu) es el telón de fondo sobre el que el microtratamiento se proyecta en las tres dimensiones peirceanas (lingüística, lógica y comunicativo-documental). Los dos primeros traen aspectos teóricos e históricos de la Semiótica documental: representación del contenido, descripción documental, operaciones y lenguajes micro-documentales y sistematización lexicográfica. El tercero porta las bases pragmáticas, teóricas y técnicas de la documentación, con seguimiento histórico que recorre la fase preotletiana, su actual estado como "ciencia normal" y propuesta de un nuevo paradigma radicado en su "extensión" mediante el concepto de interdocumento. El cuarto es desarrollo sistemático y detallado de la fórmula concepcional con la que el autor caracteriza la teoría de la documentación en el libro Concepción Lógico-Lingüística de la Documentación.

El núcleo denominado Para una teoría de la Representación Documental se propone situar la teoría y praxis de la representación de contenidos en la semiótica peirceana y aprovechar las distintas teorías de la representación semántica para realizar un análisis de contenido que sea netamente documental. El del Ensayo histórico de la Semiótica Documental pretende dar con las raíces históricas de la lingüística documental más allá de los tópicos habitualmente tratados por los investigadores de nuestra disciplina. El hilo conductor es el concepto de microdocumentación y microtratamiento de conteni- dos. La conceptualización de la semiótica documental se desarrollará en su obra Esquemas de Lingüística Documental (1990). Del tercer trabajo destacamos el concepto de "interdocumentación" (ciencia de la búsqueda secundaria) a la que se accederá una vez que se superen algunos atavismos biblioteconómicos, se acceda al archidocumento y se generalice la articulación de todos los contenidos científicos. Esta teoría del archidocumento y la Ciencia de la Búsqueda Terciaria (CB3) supuso una importante aportación a la teoría del hipertexto y del mundo de Internet. El último trabajo, que compone el artículo base analizado es el de "microdocumentación, atiende a un estudio de las "tecnologías microdocumentales y de sus aplicaciones". Arranca también del concepto de microtratamiento de contenidos y pretende acceder a la construcción de un modelo de microdocumentación, mediante el estudio de las nuevas microtecnologías y sus aplicaciones específicas al contenido menudo y pormenorizado de los entes documentales.

En la sección Pluralismo metodológico: la actitud de Feyerabend (2004) nuestro teórico de la documentación se aproxima epistemológicamente al concepto de ciencia de la documentación y a los métodos de investigación en esta área a partir de los supuestos defendidos por algunos filósofos de la ciencia, concretamente, por Karl R. Popper, Thomas Kuhn o Paul Feyerabend. Con raíces del perspectivismo orteguiano y desde una revisión a la actitud feyeberabiana en torno a la filosofía de la ciencia, defiende un pluralismo metodológico para la investigación en las ciencias de la documentación, abiertas a la pluridisciplinariedad, inter y transdisciplinariedad.

\subsection{Investigaciones adscritas a la Teoría de la Comunicación y Semiología}

Esta sección acoge un amplio trabajo que teoriza sobre la comunicación humana y los procesos de semiosis.

Sobre la trasducción (meditaciones semiológicas) I: transmisión y desustanciación (1980) constituye un modelo explicativo de los procesos y modelos comunicativos desde la dimensión semiótica y psicosociológica del mensaje, especialmente desde su dimensión pragmáticodocumental. Es un trabajo fundamentado en los procesos de génesis y transformación del discurso sumulista estudiado en la tesis doctoral del autor. Toma el modelo tópico de comunicación desde sus orígenes informáticocibernéticos. Presenta sus aplicaciones en la crítica literaria, antropología, etnología, funda- 
mentos para el procesamiento textual dentro de las tendencias del análisis del discurso actual, con indicación de las distintas opciones analítico- metodológicas que tuvo en esas disciplinas. Como contribuciones más originales se destacan: las precisiones terminológicas sobre "código" y "lengua"; la presencia del modelo en la retórica de Aristóteles; la evolución del triángulo semántico desde los estoicos hasta el más conocido de Orden-Richards; la llamada de atención sobre el contexto y la situación comunicativa y el estudio comparativo de los diversos procesos de semiosis, entrañados por los canales de la palabra y la escritura. Entre otras aportaciones colaterales destacan el estudio de la teoría del lenguaje de Ortega y Gasset, la concepción dinámica del mensaje o el análisis del concepto de intertexto. La sustitución de la metáfora de la "comunicación" por la de la "transducción" constituye el modelo reformado de comunicación, con incidencia no sólo en la teoría de la comunicación sino también en el modelo documental desde la línea de la Interdocumentación.

\subsection{Investigaciones relacionadas con la semiótica documental}

Esta serie de investigaciones aporta fundamentos teóricos para organización del conocimiento y la investigación lingüístico-documental básica. Pertenecen a dos tipos: a) una teoría general de la organización documental del conocimiento; y b) una teoría del análisis de los lenguajes naturales y las aplicaciones analíticas de las ciencias formales, con incidencia en modelos automáticos de representación de contenidos, recuperación de información y planificación textual. Su pertinencia a la lingüística documental queda justificada desde el momento en que todos ellos teorizan sobre el análisis de contenido en un sustrato metodológico básico. En esta línea, destacamos las aportaciones de la lingüística matemática y el análisis del discurso (ingeniería lingüística) que posibilitan el tratamiento automático de contenido, la aplicación de una metodología del análisis formal a la automatización de los procesos de génesis y la transformación e interpretación de productos textuales desde una perspectiva semiótico-comunicativa y digital.

\subsubsection{El marco general de la organización documental del conocimiento}

Agrupamos aquí una serie de trabajos relacionados con los aspectos paradigmáticos y epistemológicos de la organización y representación del conocimiento, desde el marco interdisciplinar. Constituyen avances en reflexiones teóricas y prácticas, fundamentos gnoseológicos, principios teoréticos y metodológicos de la organización y representación del conocimiento, etc.

La organización documental del conocimiento: marco documental/ corpus otletiano (1995)

Es la primera monografía que aborda, de un modo sistemático y crítico, el estudio del pensamiento otletiano desde la óptica actual de la organización del conocimiento. La obra se desglosa en dos extensos volúmenes interrelacionados I/1 El marco documental y l/2 El marco documental (corpus otletiano), en los que se analiza la teoría de la documentación de Paul Otlet. El objetivo del autor respecto a de re otletiana es ir mostrando otros modi res considerandi, siendo la cosa el legado de Otlet y considerada desde el punto de vista de la organización documental del conocimiento. Atiende pues a la concepción epistemológica de la documentación del bruselense y a su teoría sobre el documento.

A la luz del estudio descubrimos las dimensiones científicas de la documentación en Otlet y advertimos posicionamientos semióticos en el bruselense, claramente documentados e interpretados por Izquierdo Arroyo. Éstos se insertan dentro de la teoría del signo de Otlet, considerado desde cuatro perspectivas (cifra, notación, alfabeto e imagen), y vinculadas en un sistema gráfico universal. Destacamos también su fórmula del documento/libro como patrón integrador en el que poder basar la descripción (catalogación) y clasificación del documento así como su formulación de los elementos del documento: forma material o continente y forma intelectual o contenido. En esta distingue: elementos de redacción, disposición de los elementos y disposición del texto (fórmulas). Estos últimos constituirán la base sobre la que edificamos nuestro concepto de morfología documental de contenido y categorías retorico-funcionales de contenido (Izquierdo Alonso, 2003; 2004).

Izquierdo subraya también el concepto de relativización del contenido en Otlet en el marco de la teoría de la mnéme (p. 290-ss) y la diferenciación de perfiles de usuarios, desde la bibliopsicología y la psicosociobibliología. Se enfatiza también el uso del término matebibliología y bibliología sociométrica en el Tratado de Documentación (1934), con una interesante medición aplicada al contenido desde la dimensiones de la lecturidad, lecturabilidad y legibilidad (p. 304ss.)

No menos interesante es la aplicación de la Bibliología tecnológica y el concepto de hipertexto en Otlet, revisado en la obra que estamos reseñando. 
Organización del conocimiento / organización del conocimiento documental / organización documental del conocimiento (2003)

Es en una ponencia en el VI Congreso del Capítulo español de ISKO, celebrado en Salamanca, y en el contexto del papel de la formación teórica en la enseñanza de la organización del conocimiento, donde Izquierdo detalla más pormenorizadamente las actitudes reduccionistas respecto al término organización del conocimiento (OC) como sinónimo neto de clasificación y donde establece una clara diferencia teleológica entre la organización del conocimiento y la organización documental del conocimiento (ODC). El concepto no es nuevo, y forma parte del ideario del autor reflejado en varios de sus trabajos: Concepción lógico-lingüística de la Documentación (p. 353-361), en la obra Organización documental del conocimiento (I/1, p. 2-3, 10 y passim) o en el artículo Estructuras conceptuales para la representación documental ( $\mathrm{p}$. 29 ), entre otros. Lo diferencia de otros como organización de la documentación y organización del conocimiento y lo contextualiza en el proceso del tratamiento documental de contenido.

Según Izquierdo, hay una cierta tendencia a entender organización como sinónimo de clasificación y si bien toda clasificación es una organización, no toda organización es una clasificación. La clasificación es uno de los subprocesos de la organización pero quizás no el más importante. Hay otros que atienden a los procesos de la indización y el resumen. Así entendida para Izquierdo, la organización documental del conocimiento, equiparada con el tratamiento documental de contenido (TDC), se amplía y extiende en tres dimensiones:

- Primera dimensión: con procedimientos intermedios entre los tópicos de la indización y el resumen tales como: esquematización, integración de metadata, creación de documentos estructurados mediante diferenciación de zonas documentales, con tipos de información específica y creación de archidocumentos con vinculación hipertextual y marcado de estructuración de contenido.

- Segunda y tercera dimensión, atendiendo a la gradación de sus niveles analíticos, y concibiendo una macro, meso y microdocumentación en la ODC. Desde un nivel interdocumental a partir de la concepción del archidocumento y géneros documentales hasta un nivel intradocumental a partir del marcado de un documento como documento estructurado (lógico-retórico) y etiquetado hasta su granulación en zonas documentales de recuperación semántico-funcional especificada.
Izquierdo propone desde este posicionamiento, reformular el concepto de almacenamiento de información a partir del concepto de organización extendida (ampliada a la esquematización, la categorización el marcado y la estructuración pragmático-funcional del contenido) y desde nuevos parámetros de data mining, text mining, information extraction y summarizing.

Organización facetada del conocimiento: idea orteguiana (1999)

Se realiza una lectura crítica de los campos pragmáticos de Ortega y de la idea que éste tenía sobre la organización facetada que ha de tener el conocimiento, ponderando la doctrina del punto de vista y de la realidad como perspectiva y la integración de las facetas en el marco general y circunstancial de la vida humana. Sus aportaciones a la teoría de la organización del conocimiento, desde la perspectiva filosófica orteguiana, se enriquecen en 2004 con el estudio Vinculación onto-gnoseológica del sujetoobjeto.

Teorías formales de la representación y de la definición: taxonomía y teoría general de la clasificación

Al teorizar sobre el tratamiento semiótico y la indole epistémica de la lingüística / semiótica documental diseña un espacio diferenciado para la representación, construyendo toda una teoría de la representación documental que recoge el legado aristotélico-porfiriano, las concepciones kantianas y la línea pragmatista de Morris y Peirce. Especialmente sugestivas, como espacios para la representación documental, son sus teorías formales y semánticas de la clasificación (ELD, p. 528-ss) en las que se plantea cuestiones de la filosofía de la clasificación. Para ello, revisa críticamente conceptos, estructuras y categorías ontológicas desde Aristóteles, pasando por Porfirio, Ramón Lull, hasta Hartmann. Esta concepción extendida de la conceptualización y formalización en arborescencias, diagramas y grafos sitúa a nuestro autor en un estadio de investigación avanzada en su tiempo (fines de los 80 e inicios de los 90), al asumir nuevos modelos de representación y visualización de información para los lenguajes documentales, desde la noción de archidocumento y sus vínculos asociados, hoy técnicas aplicadas a la web semántica, y aplicando esquemas lógicoformales basados en la lógica de enunciados, lógica de clases, de relaciones y teoría de grafos para la representación de los espacios de pensamiento en términos foucaultianos.

Dedica también parte de sus reflexiones a la conceptología, desde una aproximación a la teoría de la definición en el marco de la repre- 
sentación documental $(1995,2001)$. Aplicará el término ciclo definicional en el contexto de la ordenación de conceptos- estructuras conceptuales y acuñará el término sistemas definicionales para la construcción de sistemas de representación del conocimiento, lenguajes documentales (2001).

Para completar sus contribuciones a las teorías semántico-formalistas de la organización del conocimiento, apuntamos que también realiza una lectura crítica de los campos pragmáticos de Ortega y de la idea que éste tenía sobre la organización facetada que ha de tener el conocimiento, tal y como hemos señalado anteriormente.

\subsubsection{Fundamentación científica de la Lingüística/ Semiótica Documental}

Este grupo de trabajos constituyen investigaciones generales en torno a la semiótica documental estándar, descriptiva y comparada.

Esquemas de lingüística documental (LD) (1990)

Constituye una monografía de referencia obligada en los estudios sobre análisis documental de contenido y su herramienta específica: los lenguajes documentales. En ella se asientan las bases de la concepción científica de la LD, como disciplina teórico-práctica que se plantea el almacenamiento racional y ulterior recuperación del contenido analítico de cualesquesquiera documentos (definición pragmática). En este marco, se aborda la conceptualización desde el modelo de la fórmula concepcional, aplicado también a teoría de la documentación, desde las vías correlativas de la definición y denominación. La demarcación se realiza desde dos dimensiones: la externa por lo que una disciplina queda distinguida (definida) de sus más próximas y desde la interna o partición por lo que la disciplina se estructura internamente en partes. Asimismo, se analizan pormenorizadamente los presupuestos metodológicos básicos para el tratamiento documental de contenido, desde una óptica interdisciplinar. Dicho tratamiento constituye la base de la definición semántica de la disciplina, cuyo objeto mediador es el tratamiento semiótico en la acepción promovida por Ch. Peirce, para quien la semiótica aúna, ternariamente, las perspectivas de: la gramática (aspecto lingüístico propiamente tal), la lógica formal-material (aspecto lógico) y la retórica, peculiarmente entendida desde el aspecto comunicativo y pragmático. Izquierdo, en consonancia también con las teorizaciones de Ch. Morris, señala que dicho tratamiento semiótico se mueve en la dimensión sintáctica, semántica y pragmática. Ya en 1983 nuestro autor apostaba por el término semiótica vs. lingüística: "la diferencia entre lo lingüístico y lo semiótico (o semiológico) es lo que media entre el signo lingüístico y el signo en general. Lo semiótico es más comprehensivo que lo lingüístico. (Sagredo-Izquierdo, 1983, p. 33). Consiguientemente, la semiótica documental sería una especificación disciplinar de la Semiótica general y habría de entenderse como ciencia que estudia la semiosis documental (Izquierdo, 1990, p. 64-ss).

En su sistematización expositiva de la LD como disciplina científica identifica tres aspectos: el teórico-especulativo (lingüística documental teórica), el normativo-regulador (lingüística documental normativa) y el de la praxis (lingüística documental aplicada o técnica). Atendiendo al grado de teoricidad, defiende tres dimensiones para la LD:

- Una lingüística documental general (LDG) que proporciona la teoría general para poder hablar de cualquier proceso documental en la perspectiva lingüística: naturaleza del tratamiento documental; teoría del análisis de contenido (reconocimiento-descripción-representación documental); estructura y dinámica del lenguaje documental; y teoría de la clasificación, la indización y teoría del resumen.

- Una lingüística documental descriptiva (LDD) que presenta y describe los lenguajes documentales en sus características (estructura y función propias) como sistemas semióticos y en el contexto de una gramática documental: clasificación de los lenguajes; bases lógicoformales y taxonómicas de la descripción y marco de la búsqueda documental. En esta dimensión podemos incluir también la Lingüística documental histórica (LDH) como orientación diacrónica que incluye el eje temporal y, por tanto, la evolución de los sistemas y los usos.

- Una lingüística documental comparada (LDC) en la que se comparan las estructuras y los usos de los diferentes lenguajes, entendidos como sistemas de signos.

- Una lingüística documental aplicada y normativa (LDA y LDN).

- Una lingüística documental pura (LDP) aún por construir.

De la semiótica del discurso a la semiótica documental (1993)

El trabajo justifica la adopción del ámbito interdisciplinar de las ciencias del texto para los procesos documentales de análisis de contenido desde la concepción de la semiótica como ver- 
tebradora del discurso documental. Presenta un marco epistemológico que permite situar la semiótica documental a partir de las propuestas más interesantes de las ciencias del lenguaje. Son varias las disciplinas que intervienen en el TDC. Este término se usa en sentido extenso para describir una compleja red de procesos centrados en aspectos y ámbitos diferentes en los que se produce la intersección de disciplinas tan diversas como la filosofía, las ciencias sociológicas y cognitivas, los sistemas expertos y la inteligencia artificial y las ciencias del lenguaje, entre otras.

Izquierdo reivindica la existencia de una rama general, la semiótica documental, como espacio integrador y vertebrador de los procesos de meta-representación. Desde una posición más avanzada, y a partir de posicionamientos pragmáticos, toma como punto de referencia del tratamiento documental de contenido la función del objeto semiótico (objeto en situación), determinada tanto por los elementos formales como de contenido del propio objeto, y adaptada a los objetivos de la situación comunicativodocumental. Desde estos valores semióticos, conecta con las nociones de intertextualidad, hipertextualidad y paratextualidad como espacio de meta-representación en el que los objetos semióticos son considerados como productos que forman parte del sistema de una comunidad discursiva con sus prácticas sociales (incluye aquí los esquemas epistemológicos o de dominio). Por tanto, la consideración de los determinantes contextuales es fundamental para entender la inter y paratextualidad, como mecanismo facilitador de las relaciones entre documentos-textos-símbolos en contextos interactivos de comunicación o procesos de semiosis documental.

\subsubsection{Procesamiento del lenguaje}

y gestión documental: lenguajes documentales y lingüística aplicada

Incluimos en este grupo estudios que cubren los aspectos lingüísticos aplicados al TDC, desde la concepción del lenguaje documental como un sistema semiótico a partir de tres ejes interrelacionados: a) sintáctica, semántica y pragmática del lenguaje documental, b) sintaxis y semántica estructural, y) el aspecto funcional- pragmático.

Son trabajos que atienden a las bases descriptivas de los lenguajes documentales (tipología y evolución de los mismos). Especial interés le confieren a nuestro teórico de la organización del conocimiento los lenguajes de estructura combinatoria, atendiendo a cuestiones de terminología metalingüística y precisando el alcance de vocablos como combinación, coordinación, pre y postcoordinación (1995) o desde la rama de lingüística contrastiva, comparando sistemáticamente las listas de encabezamientos de materia y los thesauri, poniendo de relieve sus semejanzas y diferencias así como sus posibilidades pragmáticas y evolutivas (1992, 1994).

\subsubsection{Modelos formales y análisis automático del discurso: procesamiento discursivo y recuperación de información}

Encuadramos en esta sección los estudios que analizan el lenguaje desde formulaciones y representaciones lógico-matemáticas. Aplicado al campo de crítica textual, y en el contexto de la semiótica y pragmática literaria, avanzó un modelo matemático para el análisis textual (1980). Englobamos también, dentro de esta faceta de procesamiento discursivo, otros trabajos como: Análisis lógico de microdiscursos naturales (1987), Elaboración de documentos "asistida" por ordenador: avance de microsistema para la organización del conocimiento (1993), y Tecnologías documentales: memorias ópticas (1994).

Los trabajos esbozan un modelo matemáticotopológico para el análisis transfrástico de los lenguajes naturales escritos. El control topológico de los contextos y entornos textuales se realiza mediante un tratamiento de los segmentos textuales que, al ser expresados mediante diagramas geométricos y arbóreos, permite representar sistemáticamente los fragmentos textuales de lenguajes escritos relativamente extensos. En los trabajos se proyecta asimismo un modelo analítico de pequeños discursos naturales que proporciona un aparato formal capaz de generar dichos discurso. Esta fijación del lenguaje simbólico adecuado, su transcripción a una estructura formal, y su expresión en un mecanismo genético-contrastivo, le han permitido a nuestro autor introducirse en el análisis y composición automático-textual.

Destacamos también en este contexto de procesamiento automático del discurso su análisis de las expresiones regulares en la recuperación y extracción de la información (2005), como categoría lingüística básica y elementos de codificación inter/intradocumental. Tras una previa caracterización, describe las herramientas de software que trabajan con expresiones regulares $\mathrm{y}$, previo cotejo de sus funciones y aplicaciones, procede a una inducción e integración modular de éstas.

\subsubsection{Otras aportaciones a la documentación}

Primera incursión en la Documentación Genealógica: realidades y sugerencias (2006) 
En este tipo de documentación especializada, Izquierdo fija el concepto de documentación genealógica (DG). Esta expresión tiene las dimensiones que el autor delimita para el término 'documentación': 1) el material consistente en el conjunto o sistema de los documentos adecuados para la genealogía; 2) el proceso con el que se documenta; y 3 ) la teoría y práctica (metodología y técnicas) sobre/de ese proceso. El autor parte de la siguiente afirmación: cuando diseñamos un árbol genealógico (con soporte informático), estamos construyendo un sistema de recuperación de información específica: la información genealógica. Por tanto, diseñamos una herramienta documental.

Ahora bien, el análisis de esa construcción conlleva tres competencias básicas de la DG: a) el papel en la recogida de los datos genealógicos (búsqueda en archivos privados y públicos convencionales; búsqueda en la Internet); b) la organización documental (catalogaciónclasificación-indización) de dichos datos genealógicos; y c) el papel en el diseño y gestión documental de sistemas de información y documentación genealógica (basados en máquina).

En contraste con tales competencias, el autor constata, como problema, que la DG no está presente en los planes de estudios universitarios. Es más, tampoco ve en esos planes la disciplina científica relativa al estudio de las relaciones de parentesco y/o familiares, a la creación y gestión de árboles genealógicos (convencionales o gestionados por ordenador). En consecuencia, habida cuenta de la necesidad de que se cumplan sus competencias, postula como objetivo la puesta en marcha social de esa disciplina y, más en concreto, la incorporación de la DG a los estudios universitarios.

Para especificar los contenidos de la DG, tenemos en cuenta su presente (realidades) y su futuro (sugerencias). Ateniéndonos a su presente, analiza primeramente los recursos genealógicos (fuentes), respecto de los cuales la función de la DG será buscar datos (en archivos e Internet) para incorporarlos al sistema. Pero el autor se centra en el sistema genealógico; considerando el árbol genealógico gestionado por ordenador, como un sistema específico de almacenamiento y recuperación de información. Como patrón de análisis, considera:

1. El proceso genealógico (los datos): agentes, acontecimientos, relaciones, espacio, tiempo, $y$ fuentes.

2. La estructura de los datos: sintaxis del lenguaje genealógico (GEDCOM, GmC, GML, etc.).
3. Las operaciones en el sistema genealógico (software). Primeramente, las operaciones constructivas (almacenamiento): la entrada de datos (teclado, importación y carga); la transformación de los datos (mantenimiento) -la adición de agente, relación o acontecimiento, mutación de agente, relación o acontecimiento, elisión de agente, relación o acontecimiento, fusión de archivos genealógicos (GEDCOM)-; y la salida de datos visualización en pantalla (listados, fichas, árboles), impresión en papel, guarda de archivo, exportación de archivo a otro formato-. En segundo lugar, las operaciones heuristicas (recuperación): búsqueda de agentes, lugares, relaciones, uniones, nacimientos, defunciones, ancestros, descendientes, etc.

4. El Software de gestión genealógica. El autor revisa diversas plataformas y hace un recuento de paquetes y programas, así como un análisis comparativo-funcional que le permita el diseño ecléctico de un nuevo software.

En su estudio, también avanza algunas sugerencias. Entre otras: 1) Las relaciones de parentesco se hacen necesarias para la sistematización de los identificadores antropónimos (términos controlados individuales) dentro de las redes semánticas de los thesauri documentales. 2) La normalización (estandarización) del lenguaje utilizado en los árboles genealógicos, merced al formato GEDCOM 5.5 (básicamente) puede tomarse como modelo para la implantación de un lenguaje formal análogo que normalice -y permita su reutilización por otros gestores-, los tesauros legibles por máquina. 3) La riqueza de presentaciones (2D y 3D) emitidas por el software de gestión de árboles genealógicos sugiere la conveniencia de hacer algo análogo con el software de gestión de tesauros y clasificaciones, en general. 4) Las relaciones de parentesco ("biológico") que vertebran la genealogía convencional admiten una aplicación analógica en las que llamaremos relaciones de parentesco "doctrinal". Habremos de construir árboles genealógicos que no representen ya las relaciones dadas a nivel de familia, sino las asimismo existentes en el marco de la academia, entre maestros y discípulos...

\section{Legado doctrinal a través del Grupo de investigación SEMIODOC}

Llegado ya este punto, y tras un denso trabajo para definir el corpus teórico-metodológico de su aportación científica, aludiremos, brevemente, al legado doctrinal del magisterio de Izquierdo en su grupo de investigación SEMIODOC 
(Semiótica Documental). Dicho grupo, entendido como comunidad de práctica investigadora y docente, se constituyó en 1991, en la Universidad de Murcia. En él participamos los firmantes de este trabajo en algunas de sus líneas temáticas de acción, sobre todo las que atienden a la semiótica documental descriptiva: nuevos modelos de tratamiento documental de contenido; diseño y gestión de lenguajes documentales; taxonomía y teoría general de la clasificación; pragmática documental de la representación/reconocimiento; gramáticas generativas aplicadas a la estructuración textual; sistemas de procesamiento discursivo: modelos y técnicas de resumen documental; y tratamiento semántico de documentos icónicos y digitales, entre otras.

Desde nuestro grupo de investigación asumimos el esquema semiótico de representación documental y concebimos la semiótica documental como una disciplina dentro de la organización y representación del conocimiento, de carácter básico-aplicado, que se ocupa de los principios, leyes, procedimientos e instrumentos para los procesos de representación y simbolización de contenidos en cualquier sistema semiótico, desde la perspectiva de la metarepresentación. Dicha finalidad viene asignada a los lenguajes documentales, en el sentido más lato o extenso del término (lenguajes de clasificación, lenguajes de indización) y la gramática textual del resumen documental.

En este sentido, dos son los constituyentes sobre los que actúa la representación para la sistematización funcional y la racionalización del contenido: las estructuras simples (conceptos) y estructuras complejas (estructuras textuales):

- Estructuras conceptuales: los conceptos y sus constituyentes en redes semánticas y sistemas conceptuales.

- Estructuras discursivo-textuales, a nivel de enunciados textuales con sus constituyentes micro, macro y superestructurales. El primero se servirá de la teoría conceptual, la teoría de la definición y las técnicas del procesamiento terminológico, entre otras. El segundo, de las teorías que sobre estructuración textual y organización discursiva desde el análisis del discurso, la lingüística textual, la ciencia cognitiva y la sociología interaccional, entre otras.

Sobre estas estructuras diseñaremos, construiremos, usaremos y evaluaremos la eficacia de nuestros lenguajes documentales como códigos y herramientas de representación documental. Todo ello, desde una triple dimensión: lógico- semántica, normativa y pragmática. El objetivo de nuestra disciplina será la representación documental para la producción de nuevo conocimiento y/o la facilitación del "acceso diferenciado/especializado" al conocimiento.

Insertada dentro del contexto de las Ciencias de la Documentación, y participando de los principios y objetivos generales que vertebran a esta Ciencia en conjunto, el TDC se posiciona próximo y complementario a la recuperación de información. Sin embargo, y extendiendo la acepción común del ADC ampliamos la naturaleza y el carácter del objeto sobre el que opera (el contenido desde su consideración semiótica), sus modelos interdisciplinares y el uso de técnicas avanzadas de análisis y explotación provenientes del campo de las ciencias formales y de la ingeniería lingüística.

Respecto a la clasificación de los estudios en TDC podemos trazar tres ejes: uno teórico, otro descriptivo y un último eje aplicado. Sin embargo, no es nítida la línea que separa uno de otro ya que se retroalimentan cíclicamente. Por último es preciso apuntar que en todas las perspectivas señaladas hay que incorporar tres nuevas facetas de análisis: la histórica (eje diacronía-sincronía), la metodológica-operativa y la metacognitiva o estratégica.

Bajo el paraguas de este esquema semiótico caracterizamos también nuestra labor investigadora a través de:

- La concepción del resumen documental, desde una perspectiva sociocognitiva, enmarcada en una dimensión semióticofuncional y comunicativa (Izquierdo Alonso y Moreno Fernández, 2009). Integramos las bases teóricas y técnicas del resumen en una perspectiva holística, de enfoque comunicativo, que engloba todas las variables semiótico-textuales, la teoría cognitiva y funcional para el estudio de los procesos, y la configuración del contexto como manifestación del acto comunicativo-documental. De esto modo, incorporamos una dimensión pragmático-comunicativa al resumen documental desde una integración del contexto (situaciones comunicativas), los procesos cognitivosociales y el objeto/texto en su dimensión social de género discursivo.

- Destacamos también la revisión crítica realizada de las propuestas y modelos de diferentes teóricos del resumen documental, lo que aporta un estado sistematizado y razonado de la situación internacional de los estudios sobre resumen documental. Señalamos como aportación la categorización de 
técnicas y enfoques en cuatro perspectivas, considerando no sólo los ejes texto-producto y proceso-actividad resumidora sino también las bases lingüísticas subyacentes: la textolingüística y el estructuralismo lingüístico, los enfoques semióticos, las orientaciones de la psicología cognitiva, o el funcionalismo sociointeraccional (Izquierdo Alonso y Moreno Fernández, 2010).

- Los presupuestos pragmáticos para el tratamiento documental de contenido, desde el análisis de género como metodología discursiva (Izquierdo Alonso 2003, 2004). Así, frente a la visión lingüístico-estructuralista y la netamente cognitivista, que han orientado la concepción y evolución de los procesos y técnicas de organización y representación documental del conocimiento, atendemos a una perspectiva pragmática aplicada al TDC, con propuestas que inciden directamente en los postulados teóricos y prácticos de la semiótica documental. En este marco, defendemos una nueva dimensión de análisis para el tratamiento documental de contenido (TDC), la forma documental del contenido según teoría hjelmsleviana, y profundizamos en el análisis de los modelos de estructuración discursiva, aplicados a la especificidad de las metodologías del análisis documental de contenido. Así, partiendo de la teoría glosemática de la estructuración del signo lingüístico y la perspectiva pragmática, fundamentada en el "análisis de género", abordamos una nueva orientación teórico-metodológica como aportación específica del análisis del discurso a la organización y representación documental del conocimiento. Esta aproximación se fundamenta en el diseño de un modelo pragmático de representación de estructuras documentales de contenido. Proponemos también la creación de una teoría general de los géneros documentales, desde una perspectiva de la semiótica funcional, como aplicación específica al metadiscurso documental de las teorías de género.

\section{Consideraciones finales}

Hemos presentado en este trabajo parte de sus ideas, las más significativas, detectando núcleos epistémicos, diferenciando componentes esenciales y levantando un arquitrabado científico en un intento de recoger y organizar sus aportaciones. Su discurso documental es denso, sazonado con desarrollos teórico-metodológicos que tienen la virtud de sugerir, implícita o explícitamente, conexiones con otros diálogos científicos y articulaciones con otros problemas planteados en otros sectores disciplinarios de la teoría de la comunicación e información e incluso de otras ciencias.

Nuestro objetivo estaba claro; difundir y hacer más comprensible el legado intelectual de José María. Sin embargo, nos queda pendiente la (re)formulación de sus teorías y modelos, poniéndolos en relación dialógica con las de otros teóricos y discursos de la documentación, a nivel nacional e internacional.

Su trayectoria y su obra, bien por su fuerte conceptualización y compleja formalización bien por su esquematismo (presentando avances e interrelaciones de ideas poco desarrolladas en sus trabajos), resultan a veces de difícil hermenéutica y requieren, consiguientemente, de análisis más profundos que, sin duda, acometeremos. Basten estas páginas como reconocimiento a su obra, desde la admiración y el cariño de quienes las firman.

\section{Referencias}

Espinosa Temiño, B.; Izquierdo Arroyo, J. M.; et al. (1994b). Tecnologías documentales: memorias ópticas. Madrid: Tecnidoc, 1994. $319 \mathrm{p}$.

Espinosa Temiño, B.; Izquierdo Arroyo, J. M.; et al. (1991). Automatización y tecnologías ópticas en Información y Documentación. // Cuadernos EUBD Complutense (Madrid). 1:1 (1991) 100.

Izquierdo Alonso, M; Moreno Fernández, L. M. (2009). El resumen documental: un reto didáctico. Madrid: ANABAD, 2009

Izquierdo Alonso, M. (2003a). El análisis de género como metodología para la organización y representación del conocimiento. // José Antonio Frías y Críspulo Travieso (eds). Tendencias de investigación en Organización del Conocimiento. Salamanca: Universidad, 2003. 747-754.

Izquierdo Alonso, M. (2003b). Procesamiento pragmático para el tratamiento documental de contenido. // Documentación de las Ciencias de la Información. 26 (2003) 181-198.

Izquierdo Alonso, M. (2004). Nuevos retos para el procesamiento de la información: la gestión de la forma documental de contenido.// Revista SCIRE, 10:1 (2004) 9-31

Izquierdo Alonso, M.; Moreno Fernández, L.M. (2010). Perspectives of the studies on document abstracting: towards an integrated review of models and theoretical approaches. // Journal of Documentation, 66: 4 (2010), 563-584.

Izquierdo Arroyo, J. M (1990). Problemas y perspectivas en la Comunicación del Saber. // Filosofía y cultura a finales del siglo XX: Actas del Congreso Internacional: IX Jornadas Andaluzas de Filosofía; Mijas, 8-9 Marzo, 1989). Mijas (Málaga): Ayuntamiento de Mijas, 1990, 71-81

Izquierdo Arroyo, J. M (1990a). La Ciencia de la Búsqueda documental Secundaria. I: Razón y misión de la CBS. // Documentación de las ciencias de la Información. 13 (1990) 87-111.

Izquierdo Arroyo, J. M (1990b). Esquemas de Lingüística Documental. Barcelona: PPU, 1990, xvi +242 p. ISBN 84-7665-733-1 (vol. 1).

Izquierdo Arroyo, J. M (1990c). Esquemas de Lingüística Documental. Barcelona: PPU, 1990, iv + 264 p. ISBN 84-7665-734-X (vol. 2) 
Izquierdo Arroyo, J. M (1990d). Esquemas de Lingüística Documental. Barcelona: PPU, 1990, vi +365 p. ISBN 84-7665-735-8 (vol. 3).

Izquierdo arroyo, J. M (1992). Cuatro trabajos en curso. // Documentación de las Ciencias de la Información. 15 (1992) 35-65.

Izquierdo Arroyo, J. M (1993a). De la Semiótica del Discurso a la Semiótica Documental. Epílogo. // Moreiro González, J. A. Aplicación de las Ciencias del Texto al resumen documental. Madrid: Univ. Carlos III; Boletín Oficial del Estado, 1993. 199-216.

Izquierdo Arroyo, J. M (1995a). Estructuras conceptuales para la representación documental. // García Marco, F. J. (Editor). Organización del Conocimiento en Sistemas de Información y Documentación, 1. (Actas del I Encuentro de ISKO-España, Madrid 4-5 Nov. 1993). Zaragoza, 1995. 27-49.

Izquierdo Arroyo, J. M (1995d). La Organización Documental del Conocimiento, I/2 El Marco documental (Corpus Otletiano). Madrid: Tecnidoc, 1995, viii + 188 p.

Izquierdo Arroyo, J. M (2001b). Sistema definicional y generación de tesauro. // VI Encuentro sobre Sistemas de Información y Documentación (Ibersid'2001): Sociedad del conocimiento y documentación digital. Universidad de Zaragoza, Facultad de Derecho. Noviembre, 2001

Izquierdo Arroyo, J. M; Moreno Fernández, L. M (1995c). Problemas de terminología metalingüística en los lenguajes documentales de estructura combinatoria. // García Marco, F. J. (ed.). Organización del Conocimiento en Sistemas de Información y Documentación, 1. (Actas del I Encuentro de ISKO-España, Madrid 4-5 Nov. 1993). Zaragoza, 1995. 51-63.

Izquierdo Arroyo, J. M; Moreno Fernández, L. M. (1992). Diseño de una base de datos de prensa, controlada por un lenguaje facetado de estructura combinatoria 'thesaurus'. //. Revista Española de Documentación Científica. 15:1 (1992) 44-63.

Izquierdo Arroyo, J. M; Moreno Fernández, L. M. (1994a). Listas de encabezamientos de materia y Thesauri en perspectiva comparada. // Documentación de las Ciencias de la Información. 17 (1994) 287-310.

Izquierdo Arroyo, J. M. (1993b). A propósito de unas Reflexiones sobre la enseñanza de la Tecnología Documental. // Documentación de las Ciencias de la Información. 16 (1993) 199-239.

Izquierdo Arroyo, J. M. (1995b). Elaboración de documentos "asistida" por ordenador: un microsistema estándar para la organización del conocimiento. // García Marco, F. J. (ed.). Organización del Conocimiento en Sistemas de Información y Documentación, 1. (Actas del I Encuentro de ISKO-España, Madrid 4-5 Nov. 1993). Zaragoza, 1995. 229-245.

Izquierdo Arroyo, J. M. (1995d). La Organización Documental del Conocimiento, I/1 El Marco documental. Madrid: Tecnidoc, 1995, xi + 502 p. (Tomo I)

Izquierdo Arroyo, J. M. (1999). La organización facetada del conocimiento: idea orteguiana. // IV Congreso ISKOEspaña. (Granada, 22 Abril, 1999).

Izquierdo Arroyo, J. M. (2001a). Ordenando conceptos por ciclos definicionales. Congreso ISKO-España: La Representación y Organización del Conocimiento: Metodologías, modelos y aplicaciones. Universidad de Alcalá, Facultad de Documentación, 25-27 Abril, 2001

Izquierdo Arroyo, J. M. (2004a). Pluralismo metodológico: la actitud de Feyerabend. // Frías Montoya, J. A.; Ríos Hilario, Ana. B. (Eds): Metodologías de Investigación en
Información y Documentación. Salamanca: Ediciones Universidad de Salamanca, 2004. 253-279.

Izquierdo Arroyo, J. M. (2004b). La vinculación ontognoseológica de sujeto-objeto en Ortega. // GonzálezSandoval Buedo, José (ed.). Figuras hispánicas: Ortega y la filosofía española. Murcia: S.F.R.M., 2004. 87-118.

Izquierdo arroyo, J. M. (2005). Las expresiones regulares en la recuperación y extracción de información: conceptos, procedimientos y aplicaciones. //Congreso: X Encuentros Internacionales sobre Sistemas de Información y Documentación. Zaragoza: Universidad, 2005. (IBERSID 2005).

Izquierdo Arroyo, J.M (1979). Aspectos topológicos del lenguaje concreto escrito: Avance de un modelo matemático para el análisis textual. // Proceso de Datos. Revista de Informática. 86 (1979) 7-25.

Izquierdo Arroyo, J.M (1980). Sobre la Transducción (Meditaciones semiológicas). I: Transmisión y Desustanciación. (Continuación). // Boletín Millares Carlo. 1:2 (1980) 323-406.

Izquierdo Arroyo, J.M (1982b). Poética filosófica y/o filosofía poética /Boletín Millares Carlo. 3:6 (1982) 499-506.

Izquierdo Arroyo, J.M (1982d). Lógica escolástica postsumulista (1550-1950). // Boletín Millares Carlo. 3:6 (1982) 337-457.

Izquierdo Arroyo, J.M (1983a). Ortega y Gasset y el problema del "apriori" de la conciencia (notas del centenario). // Revista agustiniana. 24:73 (1983) 161-228.

Izquierdo Arroyo, J.M. (1980). Sobre la Transducción (Meditaciones semiológicas). l: Transmisión y Desustanciación. // Boletín Millares Carlo (Centro Regional de la UNED en Las Palmas). 1:1 (1980) 179-218.

Izquierdo Arroyo, J.M. (1989) Aproximación al relacionismo de George Berkeley. // Philosophica Malacitana (Málaga). 2 (1989) 129-146.

Moreno Fernández, L.M; Borgoños Martínez, M.D. (2002). Teoría y práctica de la CDU. Gijón: TREA, 2002.

Moreno Fernández, L.M. (1992). Una vez más: la CDU no es un thesauri. // Documentación de las Ciencias de la Información. 15 (1992) 67-83.

Morris, Ch. (1992). Fundamentos de la teoría de los signos. Barcelona: Paidós, 1992.

Pearce, Ch. S. (1982). Obra lógico-semiótica. Alfaguara: Madrid, 1982.

Sagredo Fernández, F; Izquierdo Arroyo, J. M. (1982a). Reflexiones sobre Documento: palabra/objeto. // Boletín Millares Carlo. 3:5 (1982) 161-197.

Sagredo Fernández, F; Izquierdo Arroyo, J. M. (1982b). Análisis formal de las definiciones de Documentación. /l Boletín Millares Carlo. 3/6 (1982) 239-287.

Sagredo Fernández, F.; Izquierdo Arroyo, J. M (1989a). Análisis formal de las definiciones de Documentación. // López Yepes, J. (comp.). Fundamentos de Información y documentación. Madrid: EUDEMA, 1989. 160-200.

Sagredo Fernández, F.; Izquierdo Arroyo, J. M (1989b). La concepción ordinaria de la Ciencia de la Documentación. // López Yepes, J. (comp.). Fundamentos de Información y documentación. Madrid: EUDEMA, 1989. 53-77.

Sagredo Fernández, F.; Izquierdo Arroyo, J. M. (1983b). Concepción lógico lingüística de la Documentación. Madrid: IBERCOM, 1983.

Enviado: 2014-06-17. Segunda versión: 2014-06-21. Aceptado: 2014-06-22. 